\title{
Reversed Halo Sign-Look for the Mold Within
}

\author{
Prashant Sharma, MD, FACP and Andrea D. Beltran, PA-C \\ Department of Hospital Internal Medicine, Mayo Clinic, Rochester, MN, USA.
}

J Gen Intern Med 30(1):138

DOI: $10.1007 / \mathrm{s} 11606-014-3021-8$

(c) Society of General Internal Medicine 2014

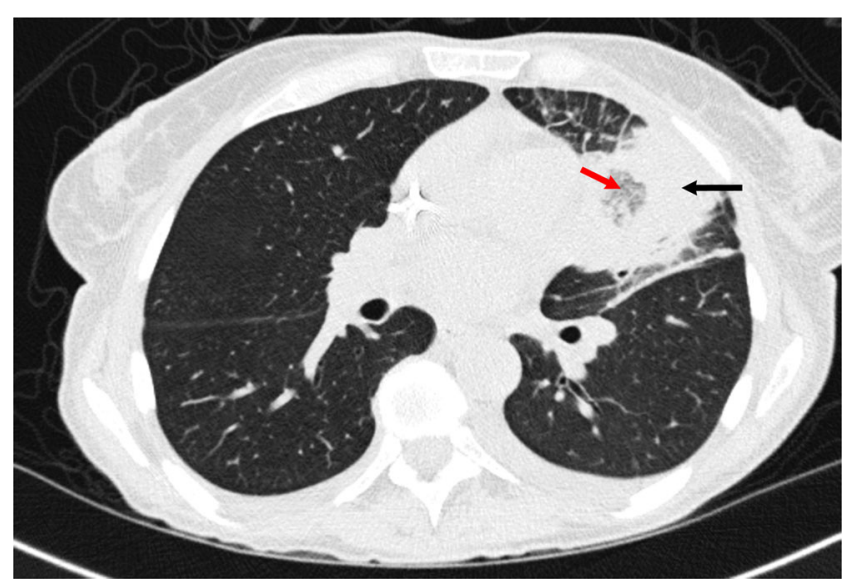

Figure 1. CT scan of the chest showing central ground glass opacity (red arrow) surrounded by a large $(6 \times 5 \mathrm{~cm})$ mass-like ring of consolidation (black arrow) representing the reversed halo sign.

\footnotetext{
A 56-year-old woman with relapsed acute myeloid leukemia on prophylactic antimicrobial agents (levofloxacin, voriconazole and acyclovir) was started on broad-spectrum antibiotics for neutropenic fevers. While blood, urine and sputum cultures remained negative, she continued to have cough and fevers. A computerized tomogram (CT) scan of the chest showed a large area of consolidation with central ground-glass opacity, the reversed halo sign (Fig. 1). Her antimicrobials were broadened to include amphotericin-B to
}

cover for mucormycosis, and caspofungin was added as adjunctive therapy. Although fungal serologies and a subsequent bronchoalveolar lavage were negative, her respiratory symptoms continued to improve on antifungal agents.

The halo sign is a CT scan finding of ground-glass opacity surrounding a pulmonary nodule or mass, while the reversed halo sign is a focal area of ground-glass opacity surrounded by a crescent or ring of consolidation. ${ }^{1,2}$ While several infectious, inflammatory and neoplastic processes can be associated with these signs, they are highly suggestive of invasive fungal infection in immunocompromised patients. ${ }^{1,2}$ A halo sign is associated with pulmonary aspergillosis while a reverse halo sign is more suggestive of mucormycosis in these patients. ${ }^{1}$ Though not pathognomonic, these signs are helpful for initiation of empiric antifungal therapy in suitable clinical settings. $^{1,2}$

Conflict of Interest: None.

Corresponding Author: Prashant Sharma, MD, FACP; Department of Hospital Internal MedicineMayo Clinic, Rochester, MN, USA (e-mail: sharma.prashant@mayo.edu).

\section{REFERENCES}

1. Georgiadou SP, Sipsas NV, Marom EM, Kontoyiannis DP. The diagnostic value of halo and reversed halo signs for invasive mold infections in compromised hosts. Clin Infect Dis. 2011;52(9):1144-1155.

2. Godoy MC, Viswanathan C, Marchiori E, et al. The reversed halo sign: update and differential diagnosis. Br J Radiol. 2012;85(1017): 1226-1235.

Published online September 30, 2014 